\title{
Estimates of the Quantum Fisher Information in the $S=1$ Anti-Ferromagnetic Heisenberg Spin Chain with Uniaxial Anisotropy
}

\author{
J. Lambert ${ }^{1,}$, and E. S. Sørensen ${ }^{1, \dagger}$ \\ ${ }^{1}$ Department of Physics \& Astronomy, McMaster University 1280 Main St. W., Hamilton ON L8S 4M1, Canada
}

(Dated: July 26, 2021)

\begin{abstract}
The quantum Fisher information is of considerable interest not only for quantum metrology but also because it is a useful entanglement measure for finite temperature mixed states. In particular, it estimates the degree to which multipartite entanglement is present. Recent results have related the quantum Fisher information to experimentally measurable probes. While in principle possible, a direct evaluation of the quantum Fisher information at finite temperatures is technically challenging and here we show that a simple estimate can be obtained for materials where the single mode approximation is valid. We focus on the $S=1$ anti-ferromagnetic Heisenberg model with uniaxial anisotropy. Quantum Monte Carlo thechniques are used to determine low temperature correlations from which the quantum Fisher information can be estimated within the single mode approximation. The quantum Fisher information is compared to the quantum variance for the staggered magnetization operators in the transverse direction and inequalities between the quantum Fisher information, the quantum variance and the full variance are discussed. Both the quantum and full variance as well as the quantum Fisher information are examined at finite temperatures above the isotropic point and at the quantum critical point for the Haldane-Néel transtion. A finite size scaling study of the quantum Fisher information is performed at the quantum critical point and used to confirm the Ising nature of the Haldane-Néel transition.
\end{abstract}

\section{INTRODUCTION}

The quantum Fisher information $(\mathrm{QFI}), F_{Q}$, is often studied in quantum metrology [1-6]. There, one considers unitary dynamics $U=\exp (-i \hat{O} \theta)$ and the phase estimation sensitivity is then limited by the Cramér-Rao bound $(\Delta \theta)^{2} \geq 1 / F_{Q}[\hat{O}]$ for any measurement. From a condensed matter perspective, the quantum Fisher information is particularly interesting since it can be used to estimate multipartite entanglement even at finite temperatures since $F_{Q} / N>m$ with $m$ a divisor of $N$ signals $(m+1)$-partite entanglement [4, 6-8]. Significant progress in the understanding of, in particular bi-partite, entanglement in quantum many-body systems has been made 9 11]. More recently, a host of techniques have been developed to efficiently quantify multipartite entanglement in quantum many-body systems. (For a review of entanglement witnesses see [12,-15]). For our purpose we will take the definition of multipartite entanglement to be the natural generalization of bipartite entanglement. Namely, consider and $N$-body quantum state $\left|\psi_{N}\right\rangle$. Now imagine expressing this state as a product of $m$ states each containing $N_{m}$ particles $\left|\psi_{N}\right\rangle=\bigotimes_{i=0}^{m}\left|\phi_{i}\right\rangle$. A $k$-partite entangled state is one for which the largest constituent state $\phi_{i}$ contains $N_{i}=k$ particles, and cannot be further decomposed. It's clear that one can recover from this the usual definition of bipartite entanglement. Ideally, for the study of multipartite entanglement, one would like to use techniques that do not rely on a particular knowledge of the density matrix, as these are the techniques most easily connected to experiment and the quantum Fisher information seem well suited for this purpose.

The quantum Fisher information (QFI) has long been

\footnotetext{
*Electronic address: lambej3@mcmaster.ca
}

${ }^{\dagger}$ Electronic address: sorensen@mcmaster.ca known as a monotonic multipartite entanglement measure [4, 6, -8], but only recently has it been connected to the dynamic structure factor which is easily accessed by experimental probes such as neutron scattering [16]. This has led to the studies of the QFI and multipartite entanglement in the Kitaev chain [17], quantum Ising chain [16], XY spin chain [18], XXZ spin chain [19] and Lipkin-Meshkov-Glick model [20, 21]. In order to access the QFI these studies all rely on the exact solvability of the models considered and from a numerical perspective, accessing the Fisher information can be challenging in particular at finite temperature for realistic non-integrable quantum many-body models. Here we show that a simple estimate of the QFI, $F_{Q}^{\mathrm{SMA}}$, can be obtained by using the single mode approximation (SMA) which allows the QFI to be calculated directly from the equal time structure factor. The quantum variance $(\mathrm{QV})$, has been established as a lower bound for $F_{Q}, F_{Q} \geq 4\left\langle\delta^{2} \hat{O}\right\rangle_{Q}$ [22] and at the same time an upper bound is given by the full variance $F_{Q} \leq 4\left\langle\delta^{2} \hat{O}\right\rangle$ [6]. This then serves as a rigorous check on the validity of the SMA calculations.

We focus on the $S=1$ AFM Heisenberg model with uniaxial anisotropy,

$$
\hat{H}=J \sum_{i}\left(\boldsymbol{S}_{i} \cdot \boldsymbol{S}_{i+1}+D\left(S_{i}^{z}\right)^{2}\right),
$$

where $D$ is the uniaxial anisotropy and we shall take $J=1$ throughout. At $D=0$ this model displays the celebrated Haldane gap at $k=\pi$ of $\Delta \sim 0.41 J$ and it is quite well established [23, 24] that the single mode approximation works very well around $k=\pi$ for moderate values of $D$. We perform stochastic series expansion [25-27] (SSE) quantum Monte Carlo simulations to evaluate low-temperature equal time correlations, from which $F_{Q}^{\text {SMA }}$ is obtained, as well as finite temperature calculations to determine the quantum and full variance. This demonstrates the presence of significant multi-partite entanglement even at the isotropic point $D=0$. 
The layout of this paper is as follows. In Section III we introduce some of the key properties of the QFI and QV (section IIA . We then introduce the single mode approximation (section [II B) and its application to the $S=1$ AFM Heisenberg model. Then in section III we present SSE results for the system QV and the QFI at the isotropic point as well as for a range of values $D<0$ towards the quantum critical point before turning to our conclusions in section IV.

\section{TECHNIQUES}

\section{A. QFI and QV}

The quantum Fisher information is one possible generalization of the classical Fisher information, which quantifies the distinguishability of a family of distributions parameterized by one (or possibly several) parameters $\theta[28,29]$. The quantum generalization of this quantifies the distinguishability of a family of quantum states defined by,

$$
\rho(\theta)=e^{-i \theta \hat{O}} \rho e^{i \theta \hat{O}},
$$

where $\hat{O}=\sum_{r} \hat{O}^{\alpha}$ is a sum over local operators. In particular, the QFI can be thought of as the statistical speed related to the rate of change of the Bures' distance, which is a metric on the space of density matrices [30]. For a density matrix that in its eigenbasis is given by:

$$
\rho=\sum_{\lambda} p_{\lambda}|\lambda\rangle\langle\lambda|
$$

the QFI is given by,

$$
F_{Q}=2 \sum_{\lambda, \lambda^{\prime}} \frac{\left(p_{\lambda}-p_{\lambda^{\prime}}\right)^{2}}{p_{\lambda}+p_{\lambda^{\prime}}}\left|\left\langle\lambda^{\prime}|\hat{O}| \lambda\right\rangle\right|^{2} .
$$

The relationship between the QFI and the multipartite entanglement has been well established in[4-8]. In particular, for a QFI density:

$$
f_{Q} \equiv F_{Q} / N>m
$$

where $m$ is a divisor of $N$, the system is $(m+1)$-partite entangled. The QFI thus increases monotonically with the entanglement. One of the most appealing features of the QFI is that it is defined for mixed states, allowing one to determine the entanglement content of a state at finite temperature. Recent work [16] has connected the QFI density to the dynamic structure factor,

$$
f_{Q}(k)=\frac{2}{N^{d} \pi} \int_{-\infty}^{\infty} \mathrm{d} \omega \tanh ^{2}\left(\frac{\omega}{2 T}\right) S(\omega, k) .
$$

The dynamic structure factor is routinely measured in inelastic neutron scattering experiments and thus provides a highly accessible measure of the multipartite entanglement of a system. In the zero temperature limit the QFI Eq. (4) reduces to the variance of the operator $\hat{O}$,

$$
F_{Q}=4\left(\left\langle\hat{O}^{2}\right\rangle-\langle\hat{O}\rangle^{2}\right)
$$

Another experimentally accessible entanglement monotone is the quantum variance[22]. The idea is that at finite temperature both thermal and quantum fluctuations contribute to the variance,

$$
\left\langle\delta^{2} \hat{O}\right\rangle \equiv\left\langle\hat{O}^{2}\right\rangle-\langle\hat{O}\rangle^{2}
$$

so that we may write

$$
\left\langle\delta^{2} \hat{O}\right\rangle=\left\langle\delta^{2} \hat{O}\right\rangle_{Q}+\left\langle\delta^{2} \hat{O}\right\rangle_{T},
$$

with the quantum fluctuations being some indicator of the extent to which a state may be entangled. In order to isolate the quantum component of the fluctuations we may use the fact that the thermal component of the fluctuations is simply given by the susceptibility. We therefore have,

$$
\left\langle\delta^{2} \hat{O}\right\rangle_{Q}=\left\langle\delta^{2} \hat{O}\right\rangle-\chi_{\hat{O}} k_{B} T .
$$

It can be shown that the QV is actually a lower bound on the QFI via the relation [22],

$$
4\left\langle\delta^{2} \hat{O}\right\rangle_{Q} \leq F_{Q}
$$

Additionaly we can see that the total variance of the operator must be an upper bound to the QFI [17]. In section III. we compute these quantities and explicitly show that they serve as a hard upper and lower bound to the single-mode approximated QFI. Both the QFI and the QV are thought to take a universal form at the quantum critical point. The exact scaling behaviour of these quantities will ultimately be inherented from the operator in terms of which they are defined.

The work in [16] derives the scaling exponents for the QFI density at both zero and finite temperature. We summarize their results here for convenience. For a review of scaling theory one can refer to [31]. Consider a rescaling of the lattice by an amount $\lambda$. The operator $\hat{O}$ will then rescale by some amount $\lambda^{-\Delta_{\alpha}}$. The QFI density will therefore scale as $\lambda^{d-2 \Delta_{\alpha}}$. Thus, we can identify $\Delta_{Q}=d-2 \Delta_{\alpha}$ as the scaling dimension for the QFI density. This result holds in the finite temperature case as well. In order to demonstrate this we recall that the temperature and frequency both scale with the dynamical critical exponent $z$. By examining Eq. (6), we see that the argument of the hyperbolic tangent function is thus scale invariant. That leaves us with the scaling of the dynamical structure factor which scales in the same was as the correlation function, and thus the finite temperature QFI will also scale as $\Delta_{Q}=d-2 \Delta_{\alpha}$. For large but finite systems at low but non-zero temperatures we then expect [16]

$$
f_{Q}(T, L)=\lambda^{\Delta_{Q}} h\left(\lambda^{z} T, \lambda / L\right)
$$

where $L$ is the linear size of the system. If simulations are performed at low enough temperatures that the scaling with $T$ can be neglected, it then follows from finite-size scaling that

$$
f_{Q}(L) \sim L^{\Delta_{Q}} .
$$




\section{B. The Single Mode Approximation}

We consider the first principles definition of the structure factor for the spectrum of the Hermitian operator $\hat{O}$ [32]

$$
S(\omega, k)=2 \pi \sum_{\lambda, \lambda^{\prime}} p_{\lambda}\left|\left\langle\lambda^{\prime}|\hat{O}| \lambda\right\rangle\right|^{2} \delta\left(\omega+E_{\lambda}-E_{\lambda^{\prime}}\right)
$$

where $p_{\lambda}=e^{\beta E_{\lambda}} / \mathcal{Z}$. The structure factor is a function of $k$ through the definition of the $\hat{O}$. In the limit of $T \rightarrow 0$ it can be shown that Eq. (14) takes on the simpler form:

$$
S(\omega, k)=\sum_{i, \lambda^{\prime}}\left|\left\langle\lambda^{\prime}|\hat{O}| 0\right\rangle_{i}\right|^{2} \delta\left(\omega+E_{0}-E_{\lambda^{\prime}}\right) .
$$

Here $|0\rangle$ is intended to represent the ground state. In general, the ground state may be degenerate. The summation index $i$ includes all states having the ground state energy $E_{0}$. The content of the single mode approximation is twofold. First we assume that only the first two energy levels are substantially populated. Second, we assume that transitions from the ground states to state at energies above the first excited state have negligible matrix elements compared with transition from the ground state manifold to the first excited state. That is to say:

$$
S(\omega, k)=S_{0}(k) \delta\left(\omega-\omega_{k}^{(01)}\right)+\tilde{S}(k, \omega),
$$

where $\left|S_{0}(k)\right| \gg\left|\int_{-\infty}^{\infty} \tilde{S}(\omega, k) \mathrm{d} \omega\right|$, and $\omega_{k}^{(01)}:=E_{1}-$ $E_{0}$. In other words, the bulk of the spectral weight is on the transition between the ground state and the first excited state. The $\widetilde{S}(\omega, k)$ represents the spectral weight coming from states above the first excited state. It is important to ask how this approximation behaves at finite temperatures where the population of excited states will increase. Let's consider the leading order correction to Eq. (14) at finite temperatures. We write the partition function as $\mathcal{Z}=$ $\sum_{i} \eta_{i} \exp \left(-\beta E_{i}\right)$, where $\eta_{i}$ denotes the degeneracy of the $i^{\text {th }}$ energy level. We may thus write the partition function as, $\mathcal{Z}=\exp \left(-\beta E_{0}\right)\left(\eta_{0}+\sum_{\lambda} \eta_{\lambda} \exp \left(-\beta \omega_{k}^{(0 \lambda)}\right)\right)$ where $\omega_{0 \lambda}$ is the energy difference between the ground sate and $|\lambda\rangle$. Substituting this into Eq. (14) and taking the temperature to be zero recovers Eq. (15). Taking instead the temperature to be small but non-zero, the next leading order correction is given by,

$$
\begin{aligned}
S(\omega, k)= & \frac{\delta\left(\omega-\omega_{k}^{(01)}\right)+\delta\left(\omega+\omega_{k}^{(01)}\right) e^{-\beta \omega_{k}^{(01)}}}{\eta_{0}+\eta_{1} e^{-\beta \omega_{k}^{(01)}}} S_{0}(k) \\
& +\tilde{S}(\omega, k) .
\end{aligned}
$$

Not surprisingly, the next leading order correction is spectral weight coming from transition from the first excited state to the ground state. In general when integrated over, this thermal correction will manifest as a multiplicative factor of the form,

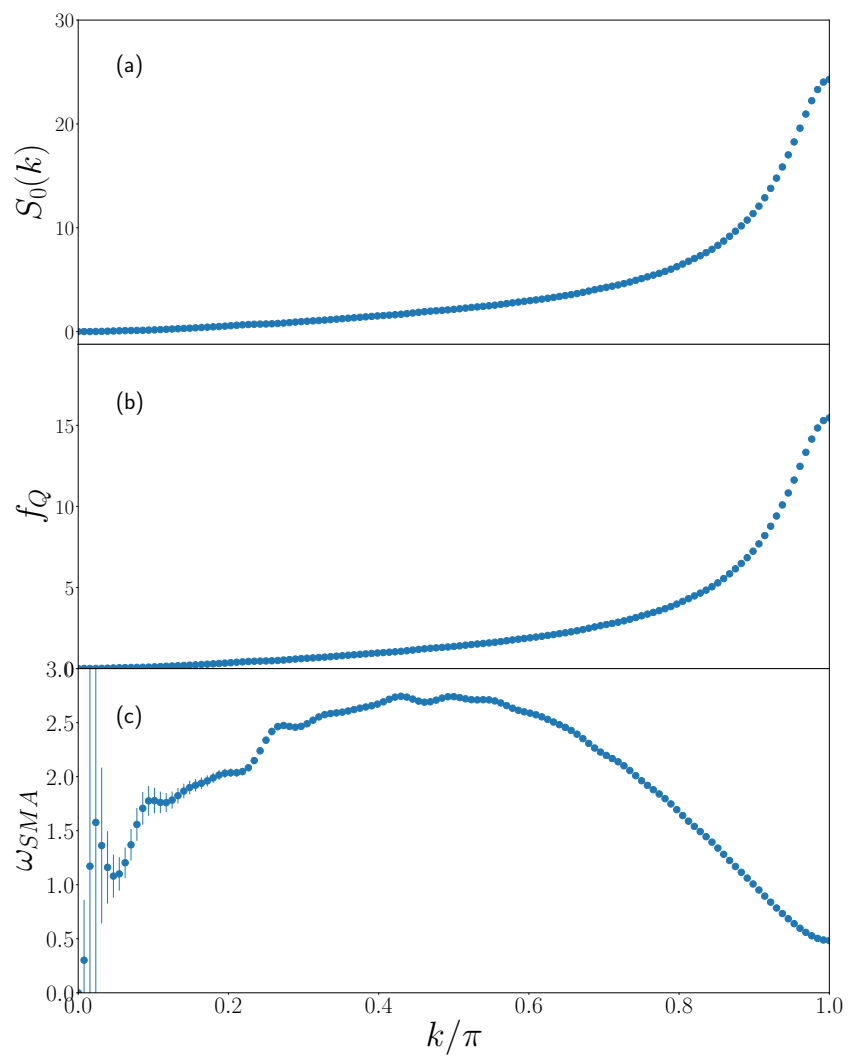

Figure 1: (Color online) (a) The equal time structure factor for the isotropic $S=1$ AFM model with $N=256, \beta=400$, exhibiting at peak at $k=\pi$. (b) The QFI density in the first Brillouin zone approaching zero at $k=0$, and exhibiting a peak at $k=\pi$. (c) $\omega_{k}$ as obtained from the single-mode approximation. The characteristic gap of $0.41 J$ at $k=\pi$ is clearly visible.

$$
A=\frac{1+e^{-\beta \omega_{k}^{(01)}}}{\eta_{0}+\eta_{1} e^{-\beta \omega_{k}^{(01)}}} .
$$

When the ground state and excited state are non-degenerate, this factor will be equal to unity, and therefore will not affect the quantum Fisher information. For a more thorough examination of the effects of temperature on the entanglement near a critical point see [41]. The approach there is effecitively a single-mode approximation but applied directly to the QFI and used to examine integrable systems.

In order to employ the single mode approximation we need some way to determine the gap, $\omega_{k}^{(01)}$ (we henceforth drop the superscript and allow $\omega_{k}$ to denote the dispersion for the first excited state). It is clear that due to energy conservation $\operatorname{supp}(\tilde{S})=\left\{\omega: \omega_{k}<\omega_{c}<\omega\right\}$, where $\omega_{c}$ denotes the bottom of the continuous portion of the energy spectrum. In order to determine $\omega_{k}$ we multiply Eq. (16) by $\omega$ and integrate over all frequencies:

$$
\frac{1}{\omega_{k}} \int_{-\infty}^{\infty} \mathrm{d} \omega \omega S(\omega, k)=S_{0}(k)+\frac{1}{\omega_{k}} \int_{-\infty}^{\infty} \mathrm{d} \omega \omega \tilde{S}(\omega, k) .
$$


In order to deal with the $\tilde{S}$, we note that:

$$
\frac{1}{\omega_{k}} \int_{-\infty}^{\infty} \mathrm{d} \omega \omega \tilde{S}(\omega, k) \geq \int_{-\infty}^{\infty} \mathrm{d} \omega \tilde{S}(\omega, k) .
$$

This assertion is made valid by the positive semi-definite nature of $\tilde{S}$. By substituting this inequality into Eq. (19) we see that the LHS is, by definition, the equal time structure factor $S(k)$, giving:

$$
\frac{1}{S(k)} \int_{-\infty}^{\infty} \mathrm{d} \omega \omega S(\omega, k) \geq \omega_{k}
$$

with

$$
S(k) \equiv \int S(k, \omega) d \omega=\sum_{r} e^{-i k r}\langle S(r) \cdot S(0)\rangle .
$$

We may use the following sum rule [33]:

$$
\int_{-\infty}^{\infty} \mathrm{d} \omega \omega S(\omega, k)=\pi\left\langle\left[\hat{O}^{\dagger},[H, \hat{O}]\right]\right\rangle,
$$

to evaluate this expression, which leaves the bound on $\omega_{k}$ as:

$$
\omega_{k} \leq \omega_{\mathrm{SMA}}(k):=\pi \frac{\left\langle\left[\hat{O}^{\dagger},[H, \hat{O}]\right]\right\rangle}{S(k)} .
$$

Here, $S(k)$ along with the different components of the commutator can relatively easily be estimated using quantum Monte Carlo methods from which $\omega_{\text {SMA }}(k)$ can then be obtained. Results are shown in Fig. 1 (a) for the $S(k)$ from Fourier transforms of the ground-state correlation functions obtained from QMC calculations. Fig. 1. (c) shows the resulting $\omega_{S M A}(k)$.

\section{RESULTS}

We now turn to a discussion of our results for the QFI and multipartite entanglement in the $S=1$ AFM Heisenberg model with uniaxial anisotropy,

$$
\hat{H}=J \sum_{i}\left(\boldsymbol{S}_{i} \cdot \boldsymbol{S}_{i+1}+D\left(S_{i}^{z}\right)^{2}\right)
$$

This model has several appealing feature to investigate multipartite entanglement. First, it possesses a symmetry-protected topologically (SPT) phase with a gapped ground state in the isotropic region, with $\Delta \approx 0.41 J[34$, 35]. This phase is characterized by the breaking of a hidden $\mathbb{Z}_{2} \times \mathbb{Z}_{2}$ symmetry which establishes a long-range string order [36]. Second, the uniaxial anisotropy can drive two quantum phases transitions with critical points falling into two different universality classes. The phase diagram of this model has been extensively investigated in [37-39]. The first transition is from the topologically protected Haldane phase to a disordered phase with quasi-Néel ordering $\left(D_{C}^{H N} \approx-0.31\right)$. The second transition is to a phase which is often called the "large-D phase" $\left(D_{C}^{H L} \approx 0.98\right)$. This latter phase is essentially "empty" as

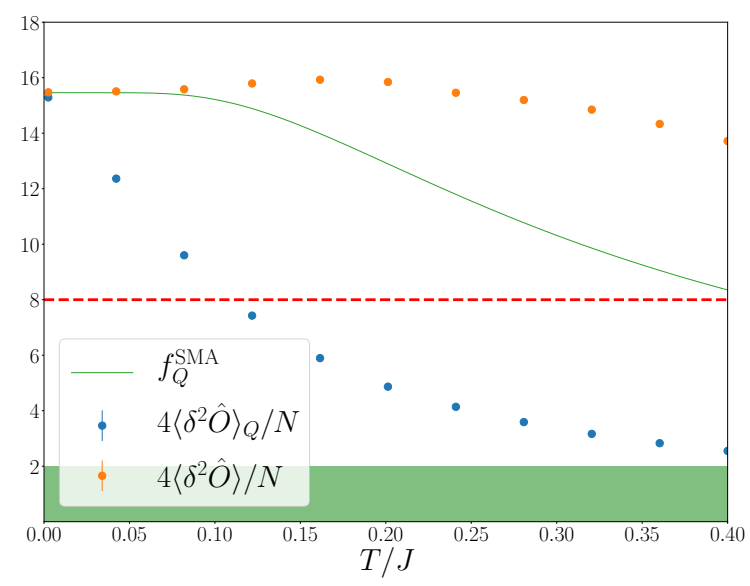

Figure 2: Finite temperature behaviour of the QFI density, $f_{Q}(k=$ $\pi)$ and the quantum variance for temperatures up to the Haldane gap at the isotropic point, $D=0$, for $N=256 . f_{Q}^{\mathrm{SMA}}$ is obtained from simulations at $\beta=400$. Upper and lower bounds for $f_{Q}$ given by $4\left\langle\delta^{2} \hat{O}\right\rangle / N$ and $4\left\langle\delta^{2} \hat{O}\right\rangle_{Q} / N$ are also shown for a range of temperatures. The green shaded region indicates the level the $f_{Q}$ has to exceed to indicate the presence of more than bi-partite entanglement. The dashed red line indicates the threshold for $(8+1)$-partite entanglement. Below that line and above the green region the system would be (4+1)-partite entangled.

the large unixial anisotropy forces each spin to have zero $S^{z}$ projection. The Haldane-Néel transition is in the universality class of the 2D Ising model, while the Haldane-Empty transition is in the Gaussian universality class. The excitation spectrum exactly at the isotropic point consists of a triplet state. This degeneracy is lifted away from the isotropic point into a heavier magnon with energy $\omega_{k}^{(\|)}$and a lighter doublet with $\omega_{k}^{(\perp)}$. This notation is meant to evoke the fact that the heavier magnon is in the direction parallel to the uniaxial anisotropy, while the doublet corresponds to the transverse excitations. Most importantly, in a sizable region around $k=\pi$, as well as for $D \neq 0$, the dynamical strucure factor is well approximated by a single mode.

We use stochastic series expansion [25, 27, 40] (SSE) techqniques to numerically study the QFI within the single mode approximation. All of the SSE simulations used in this section use on the order of $10^{6}$ Monte Carlo sweeps. The data for each obersvable is binned into groups of 1000 with the error bars estimated by taking the average variance over the bins.

In order to examine the quantum Fisher information we consider the operator, $\hat{O}=\sum_{r} e^{i k r} \hat{S}_{r}^{z}$. The equal time structure factor for this operator corresponds to the spectrum of spontaneous fluctuations in the longitudinal channel. Using Eq. 24) we may compute the bound on the dispersion for the heavy magnon to be [23],

$$
\omega_{\mathrm{SMA}}=\frac{J\left(C_{x x}^{r, r+1}+C_{y y}^{r, r+1}\right)(1-\cos (k))}{S_{0}(k)},
$$




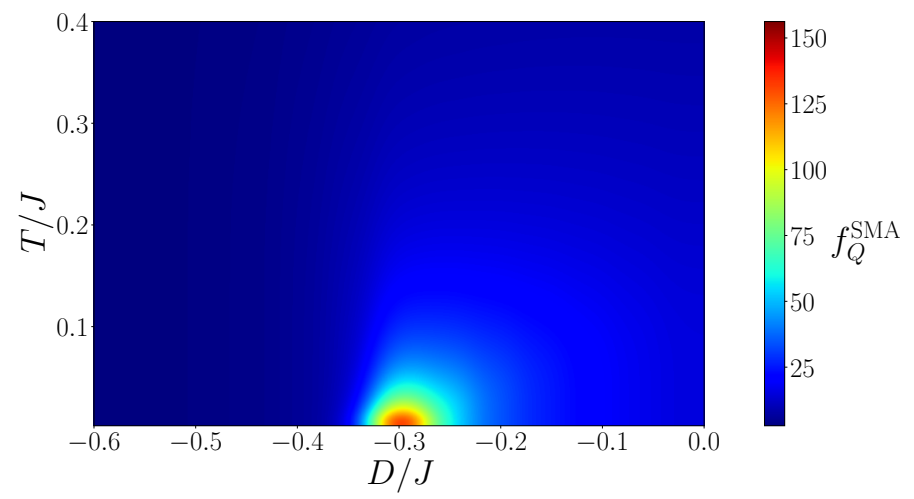

Figure 3: QFI density detected for $\hat{O}=\sum_{r} e^{i k r} S_{r}^{z}$ around the phase transition from the isotropic Haldane phase to the anti ferromagnetic phase for $\mathrm{N}=256$. Obtained from SSE results $(\beta=400)$ and periodic boundary conditions

where $C_{\alpha \beta}^{i j}:=\left\langle\hat{S}_{i}^{\alpha} \hat{S}_{j}^{\beta}\right\rangle$. For the case of periodic boundary conditions the ground state is not degenerate. We are here concerned with the singlet heavy magnon state. In this case $\eta_{0}=\eta_{1}=1$ and thus, as per equation (18), the leading thermal correction does not effect the QFI. Since the single mode approximation assumes these contributions to be small we ignore these thermal corrections. We may now apply the single-mode approximation to compute the QFI density,

$f_{Q}(k)=4 \tanh ^{2}\left(\frac{\omega_{k}}{2 T}\right) S_{0}(k)+\int_{-\infty}^{\infty} \mathrm{d} \omega \tanh ^{2}\left(\frac{\omega}{2 T}\right) \tilde{S}(\omega, k)$,

where we shall neglect the last term arising from the continuum contribution. Since this last term corresponds to a positive contribution we would expect to obtain a lower bound on the QFI. We argue, however, that the dominant effect, particularly near the isotropic point, will come from the inequality, $\omega \leq \omega_{S M A}$, Eq. (24). Hence, we believe that an overestimation of the QFI density is the more likely scenario. However, we expect this approximation to be rather good at low temperatures close to the isotropic point, $D=0$, where we then obtain the estimate for $f_{Q}$,

$$
f_{Q}^{\mathrm{SMA}}(k) \sim 4 \tanh ^{2}\left(\frac{\omega_{\mathrm{SMA}}}{2 T}\right) S_{0}(k) .
$$

We also note that the main $T$-dependence of $f_{Q}^{\mathrm{SMA}}(k)$ is now through the argument of the tanh.

Near the critical point it is expected that the continuum will contribute more significantly to the behaviour of the system especially at non-zero temperature, since the excitation gap closes. The finite temperature results at the critical point are therefore less reliable than those at the isotropic point, where the population is mainly concentrated in the ground state until temperatures of the order of half the gap $(T \sim 0.2 J)$ are reached. With anti-ferromagnetic exchange, the equal time structure factor peaks at the $k=\pi$ mode (Fig. 11)(a). Thus the quantum Fisher information is maximal at the edge of the first Brillouin zone as shown in Fig. 1 b) where $f_{Q}^{\mathrm{SMA}}(k)$ is shown throughout Brillouin zone. This corresponds to parameterizing the path through the space of density matrices

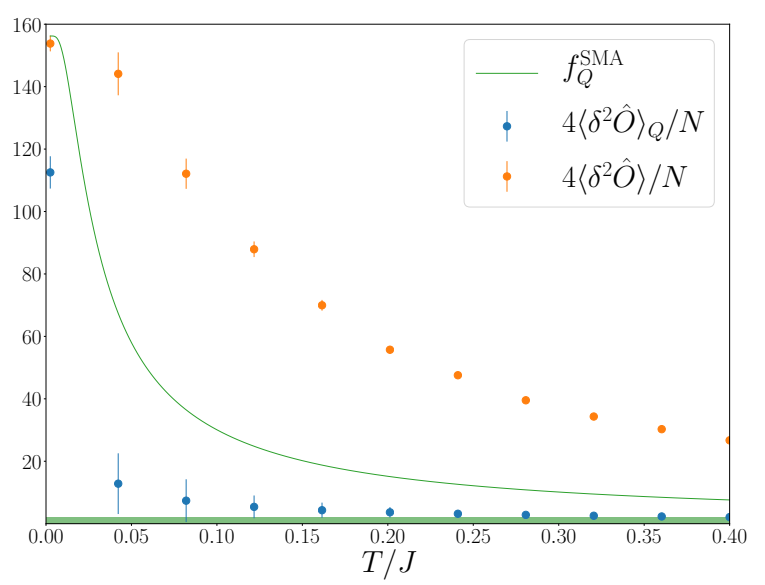

Figure 4: Finite temperature QFI density and QV above the critical point for $N=256$. $f_{Q}^{\mathrm{SMA}}$ is obtained from simulations at $\beta=400$. Upper and lower bounds for $f_{Q}$ given by $4\left\langle\delta^{2} \hat{O}\right\rangle / N$ and $4\left\langle\delta^{2} \hat{O}\right\rangle_{Q} / N$ are also shown for a range of temperatures. The green shaded region indicates the threshold $f_{Q}$ has to exceed for bi-partite entanglement to be present.

using the staggered magnetization. At $k=0, \hat{O}$ becomes the total magnetization which commutes with the Hamiltonian and thus cannot detect entanglement. As we approach $k=0$ the single mode approximation also becomes invalid since it is known that the well-defined single mode present around $k=\pi$ merges into the continuum. Fortunately the behaviour of the single mode approximation remains well controlled at the edge of the Brillouin zone where the QFI density detected by momentum space magnetization is maximal. In the following we therefore exclusively focus on $k=\pi$.

Let us first consider the finite temperature behaviour of the entanglement at the isotropic point, $D=0$. Using the $\mathrm{QV}$ we have established a lower bound on the QFI density. On the other hand, there is a well-known upper bound [6] on $f_{Q}$ as well: $f_{Q} \leq 4\left\langle\delta^{2} \hat{O}\right\rangle / N$, where $\left\langle\delta^{2} \hat{O}\right\rangle$ refers to the total variance, Eq. (8). Combining this with Eq. (28) we then obtain:

$$
4\left\langle\delta^{2} \hat{O}\right\rangle_{Q} \leq F_{Q} \sim F_{Q}^{\mathrm{SMA}} \leq 4\left\langle\delta^{2} \hat{O}\right\rangle .
$$

In Fig. 2 are shown results for $f_{Q}^{\mathrm{SMA}}(k=\pi)$ for a range of temperatures. In this regime the single-mode approximation should work quite well up until approximately half the gap. Indeed, $f_{Q}^{\text {SMA }}$ is clearly within the hard upper and lower bounds on $f_{Q}$ given by $4\left\langle\delta^{2} \hat{O}\right\rangle / N$ and $4\left\langle\delta^{2} \hat{O}\right\rangle_{Q} / N$ for all temperatures shown. We see that up until this point the approximated QFI density predicts the presence of multipartite entanglement well into this regime. If we use the quantum variance as a lower bound on $f_{Q}(k)$ then it predicts multipartite entanglement to temperatures approaching the gap. In Fig. 2 the shaded green region indicates the threshold to be exceeded for bi-partite to be present and we note that both estimates of $f_{Q}(k=\pi)$ indicate the presence of bi-partite 


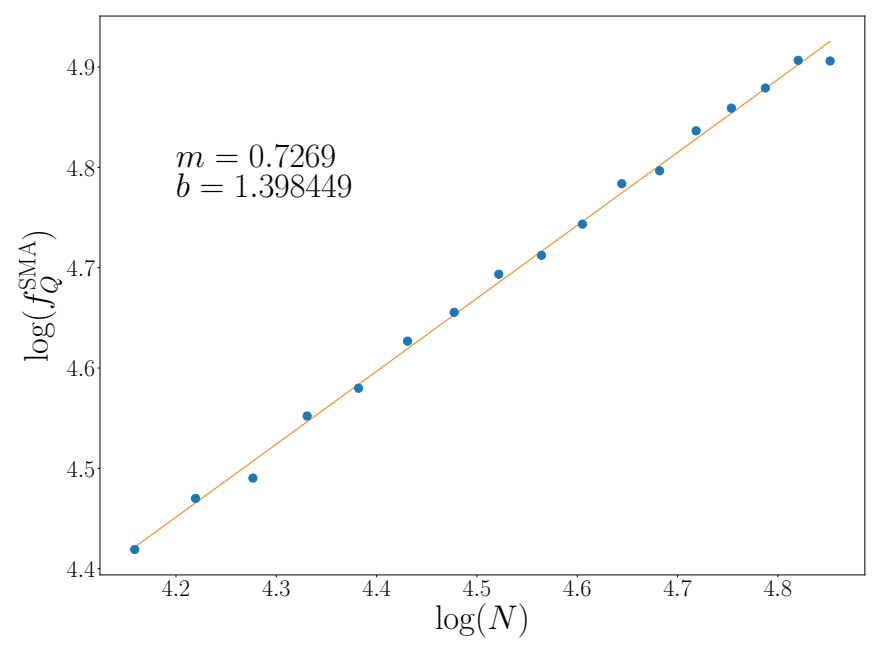

Figure 5: Finite size scaling of the QFI density with system size for even system sizes between $N=64$ and $N=128$. Scaling was performed at $D=D_{C}=-0.31$ at $\beta=400$ small enough that the system size would be the relevant perturbation to the scaling. The critical exponent with error due to the fit is found to be $\Delta_{Q}=$ $0.7269(1)$ with purely statistical error estimate.

entanglement up to temperatures close to the gap.

We may now ask how the ground state QFI density will behave as we approach the quantum critical point. Fig. (3) show the QFI density indicated by the color intensity for a range of temperatures and $D$ values for a system size of 256 and $\beta=400$ with periodic boundary conditions. We see that the QFI density is divergent at the quantum critical point, as expected from the behaviour of $\hat{O}$. Fig. (4) clearly shows the The QFI density predicted by the single mode approximation decays rapidly above the critical point, as the gap has now effectively closed. Perhaps surprisingly, $f_{Q}^{\text {SMA }}$ is also here clearly withing the hard upper and lower bounds on $f_{Q}$ given by $4\left\langle\delta^{2} \hat{O}\right\rangle / N$ and $4\left\langle\delta^{2} \hat{O}\right\rangle_{Q} / N$ for all temperatures shown. We see in this case that there is still persistent multipartite entanglement at finite temperatures above the quantum critical point.

The divergence of the entanglement at the critical point is seen by examining the QFI density for various systems sizes. Fig. 5 demonstrate the divergent scaling of both the QFI and the QV. Due to the fact that the Haldane-Néel transition is in the Ising universality class we can compute theoretically what the finite size scaling of the QFI density at the critical point must be. At low enough temperatures this is for finite systems given by Eq. (13). For the Ising universality class the critical exponent for the staggered magnetization is given by $\Delta_{\alpha}=1 / 8$. This is confirmed in [38] using cluster ex- pansion methods. This should give a QFI density scaling of $\Delta_{Q}=3 / 4$. By examining even system sizes between $N=64$ and $N=128$ at a $\beta=400$ at a value of $D_{C}^{H N}=-0.31$ we estimate a QFI density scaling of $\Delta_{Q}=0.7269(1)$. The error quoted here is associated with the quality of the linear regression. It does not account for systematic errors in the measurement of the QFI density. In order to estimate these systematic errors we examine subsets of four points and determine the maximum and minimum slopes that could be inferred from such four point subset of the data. Using this we estimate a deviation of at least \pm 0.06 . Thus the estimated scaling is $\Delta_{Q}=0.73 \pm 0.06$. This estimate is consistent with the Ising universality class predicted for the HaldaneNéel transition which is surprising since we would not expect the single-mode approximation to give reliable results at the quantum critical point.

\section{CONCLUSIONS}

Using the single mode approximation we have shown that it is possible to obtain a quite simple estimate of the QFI density that should yield reliable results at temperatures well below the gap. We studied the $S=1$ anti-ferromagnetic spin chain with uniaxial anisotropy within this approximation. The approximation yields results that are within rigoruous upper and lower bounds for all tempertures studied. Clear signatures of multipartite entanglement were found at the isotropic point, $D=0$ with the QFI density diverging when approaching the quantum critical point. When combined with the QV, the single-mode approximated QFI allows one to place both upper and lower bounds on the finite temperature entanglement of gapped systems. More precise techniques for calculating the QFI density at finite temperatures in strongly correlated systems would clearly be very desirable. Alternatively, sharper lower or upper bounds on the QFI density than we have discussed here would be very valuable.

We also note that the QFI has been linked to the canonical energy in gravitational physics [42] and can be expressed in terms of the relative entropy [43], developments which could potentially be exploited for more efficient numerical calculations of the QFI.

\section{ACKNOWLEDGEMENTS}

This research was supported by NSERC and enabled in part by support provided by (SHARCNET) (www.sharcnet.ca) and Compute/Calcul Canada (www.computecanada.ca).
[1] D. Petz and C. Sudr, Journal of Mathematical Physics 37, 2662 (1996), https://doi.org/10.1063/1.531535.

[2] D. Petz, Journal of Physics A: Mathematical and General 35, 929 (2002).
[3] M. G. A. Paris, International Journal of Quantum Information 07, 125 (2009).

[4] G. Tóth, Physical Review A 85, 022322 (2012).

[5] G. Tóth and D. Petz, Phys. Rev. A 87, 032324 (2013). 
[6] G. Tóth and I. Apellaniz, Journal of Physics A: Mathematical and Theoretical 47, 424006 (2014).

[7] L. Pezzé and A. Smerzi, Physical Review Letters 102, 100401 (2009).

[8] P. Hyllus, W. Laskowski, R. Krischek, C. Schwemmer, W. Wieczorek, H. Weinfurter, L. Pezzé, and A. Smerzi, Physical Review A 85, 022321 (2012).

[9] J. Eisert, M. Cramer, and M. B. Plenio, Reviews of Modern Physics 82, 277 (2010).

[10] N. Laflorencie, Physics Reports 646, 1 (2016), ISSN 03701573, quantum entanglement in condensed matter systems.

[11] M. Headrick, Physical Review D 82, 126010 (2010).

[12] R. Horodecki, P. Horodecki, M. Horodecki, and K. Horodecki, Reviews of modern physics 81, 865 (2009).

[13] V. Vedral, M. B. Plenio, M. A. Rippin, and P. L. Knight, Physical Review Letters 78, 2275 (1997).

[14] L. Amico, R. Fazio, A. Osterloh, and V. Vedral, Reviews of modern physics 80, 517 (2008).

[15] M. B. Plenio and S. Virmani, arXiv preprint quant-ph/0504163 (2005).

[16] P. Hauke, L. Tagliacozzo, and P. Zoller, Nat Phys 12 (2016).

[17] L. Pezzè, M. Gabbrielli, L. Lepori, and A. Smerzi, Phys. Rev. Lett. 119, 250401 (2017).

[18] W.-F. Liu, J. Ma, and X. Wang, Journal of Physics A: Mathematical and Theoretical 46, 045302 (2013).

[19] Q. Zheng, Y. Yao, and X.-W. Xu, Communications in Theoretical Physics 63, 279 (2015).

[20] S.-S. Li, H.-G. Yi, and R.-H. Chen, International Journal of Theoretical Physics 52, 1175 (2013), ISSN 1572-9575.

[21] J. Ma and X. Wang, Phys. Rev. A 80, 012318 (2009).

[22] I. Frérot and T. Roscilde, Physical Review B 94, 075121 (2016).

[23] E. S. Sørensen and I. Affleck, Physical Review B 49, 15771 (1994).

[24] O. Golinelli, T. Jolicœur, and E. Sørensen, The European Physical Journal B - Condensed Matter and Complex Systems 11,
199 (1999), ISSN 1434-6036.

[25] A. W. Sandvik and J. Kurkijärvi, Physical Review B 43, 5950 (1991).

[26] A. W. Sandvik, Physical Review B 57, 10287 (1998).

[27] O. F. Syljuåsen and A. W. Sandvik, Phys. Rev. E 66, 046701 (2002).

[28] W. K. Wootters, Physical Review D 23, 357 (1981).

[29] S. L. Braunstein and C. M. Caves, Physical Review Letters 72, 3439 (1994).

[30] M. Gessner and A. Smerzi, arXiv preprint arXiv:1712.04661 (2017).

[31] J. Cardy, Scaling and renormalization in statistical physics, vol. 5 (Cambridge university press, 1996).

[32] S. Lovesey, Condensed Matter Physics: Dynamic Correlations, Frontiers in Physics (Benjamin/Cummings, Advanced Book Program, 1980), ISBN 9780805366105.

[33] P. Hohenberg and W. Brinkman, Physical Review B 10, 128 (1974).

[34] F. Haldane, Physical Review Letters 50, 1153 (1983).

[35] I. Affleck, Physical Review B 41, 6697 (1990).

[36] T. Kennedy and H. Tasaki, Physical review b 45, 304 (1992).

[37] Z. Zhang, K. Wierschem, I. Yap, Y. Kato, C. D. Batista, and P. Sengupta, Physical Review B 87, 174405 (2013).

[38] A. F. Albuquerque, C. J. Hamer, and J. Oitmaa, Physical Review B 79, 054412 (2009).

[39] W. Chen, K. Hida, and B. Sanctuary, Physical Review B 67, 104401 (2003).

[40] A. W. Sandvik, Physical Review B 59, R14157 (1999).

[41] M. Gabbrielli, A. Smerzi, and L. Pezzè, arXiv preprint arXiv:1805.03139 (2018).

[42] N. Lashkari and M. Van Raamsdonk, Journal of High Energy Physics 2016, 153 (2016).

[43] M. van Raamsdonk, arXiv.org (2016), 1609.00026v1. 